\title{
Chain Dimensions of Polystyrene in Cyclohexane around the $\Theta$ Temperature
}

\author{
Tomo-o OYAMA, Kohzoh SHIOKAWA, and Koumei BABA \\ Department of Applied Science, Faculty of Engineering, Kyushu University, \\ Hakozaki, Higashi-ku, Fukuoka 812, Japan.
}

(Received August 5, 1980)

\author{
KEY WORDS Chain Dimension / Polystyrene / $\Theta$ Temperature / Coil- \\ Globule Transition /
}

Recent theories ${ }^{1,2}$ have predicted the following limiting relations for the chain dimensions $\left\langle S^{2}\right\rangle$ in dilute solutions.

$$
\begin{aligned}
& \left\langle S^{2}\right\rangle \sim M^{6 / 5} \tau^{2 / 5} \quad \text { (above } \Theta \text { region) } \\
& \left\langle S^{2}\right\rangle_{\Theta} \sim M \quad(\Theta \text { region) } \\
& \left\langle S^{2}\right\rangle \sim M^{2 / 3}(-\tau)^{-2 / 3} \text { (below } \Theta \text { region) }
\end{aligned}
$$

where $M$ is the molecular weight of the solute polymer and $\tau$ is the reduced temperature defined by $\tau=(T-\Theta) / \Theta(T$, the observed temperature; $\Theta$, the $\Theta$ temperature of the solution).

Equations 1 and 2 have been already confirmed by many experiments. However, the confirmation of eq 3 is still incomplete. Several experiments ${ }^{3-6}$ on $\left\langle S^{2}\right\rangle$ below the $\Theta$ temperature are concerned only with the temperature dependence of $\left\langle S^{2}\right\rangle$. For example, Nierlich et $a l^{6}$ obtained the relation $\left\langle S^{2}\right\rangle^{1 / 2} \propto(-\tau)^{-0.32}$ by small-angle neutron scattering. This result is consistent with the $\tau$ part of eq 3 . No experiments on the $M$ dependence of $\left\langle S^{2}\right\rangle$ below the $\Theta$ temperature have been reported. Recently, Bauer and Ullman ${ }^{7}$ measured the hydrodynamic radius $R_{\mathrm{H}}$ by dynamic light scattering and derived the relation $R_{\mathrm{H}} \propto M^{0.446}(-\tau)^{-0.108}$. But their derivation of $\left\langle S^{2}\right\rangle$ from $R_{\mathrm{H}}$ is somewhat obscure.

The main reason for the lack of data for the $M$ dependence is the difficulty created by experimental factors. In light-scattering experiments, high $M$ samples are favorable for the $\left\langle S^{2}\right\rangle$ measurement, but are not so for the measurable temperature range below the $\Theta$ temperature, since the precipitation temperature approaches the $\Theta$ temperature as $M$ increases. For this reason, measurement over a sufficient wide range of $M$ is impossible.

The present authors have developed a new lightscattering instrument, ${ }^{8}$ SARD, by which $\left\langle S^{2}\right\rangle^{1 / 2}$ down to $c a$. $5 \mathrm{~nm}$ can be measured. In the following, the data for $\left\langle S^{2}\right\rangle v s$. $M$ and $\tau$ above and below the $\Theta$ temperature determined by SARD and the confirmation of the $M$ part of eq 3 are reported.

\section{EXPERIMENTAL}

The samples used are "monodisperse" standard polystyrenes prepared by Toyo Soda Manufacturing Co., Ltd. The molecular characteristics of the samples are shown in Table $I$, in which the values of the weight-average molecular weight $M_{w}$ and its ratio to the number-average molecular weight $M_{n}$ were given by the supplier. $\left\langle S^{2}\right\rangle_{\Theta}{ }^{1 / 2}$ is the radius of gyration in cyclohexane at the $\Theta$ temperature, $34.5^{\circ} \mathrm{C}$. The samples were used as supplied. The solvent, cyclohexane, was purified by the usual

Table I. Characteristic data for the polystyrene samples

\begin{tabular}{lccc}
\hline Sample & $M_{w} \times 10^{-4 \mathrm{a}}$ & $M_{w} / M_{n}^{\mathrm{a}}$ & $\left\langle S^{2}\right\rangle_{\Theta}^{1 / 2} / \mathrm{nm}$ \\
\hline F-20 & 18.6 & 1.07 & 12.7 \\
F-40 & 42.2 & 1.04 & 18.5 \\
F-80 & 77.5 & 1.01 & 26.0 \\
F-126 & 126 & 1.05 & 31.8 \\
\hline
\end{tabular}

${ }^{\text {a }}$ Data given by supplier. 
method. The solvent and solutions were made clean by filtration through an $0.45 \mu \mathrm{m}$ membrane filter (Toyo Roshi Co., Ltd. TM-2P).

Light-scattering measurements were made by SARD ${ }^{8}$ After the solutions had been warmed to $60^{\circ} \mathrm{C}$, they were cooled at a rate of $0.1^{\circ} \mathrm{C} \mathrm{min}-1$ and were studied at several temperatures above their precipitation temperatures.

\section{RESULTS AND DISCUSSION}

The temperature dependence of $\alpha_{\mathrm{s}}^{2} \quad\left(=\left\langle S^{2}\right\rangle /\right.$ $\left.\left\langle S^{2}\right\rangle_{\Theta}\right)$ and the double-logarithmic plot of $\alpha_{\mathrm{s}}^{2} v s$. $M_{w}$ are shown in Figures 1 and 2, respectively. The temperature dependence of the exponent $2 v$ in the relation $\left\langle S^{2}\right\rangle \propto M_{w}^{2 v}$, obtained from Fiure 2, is shown in Figure 3.

The value of $2 v$ increases smoothly with temperature and exhibits no sudden change. However, even at the highest temperature $\left(54.3^{\circ} \mathrm{C}\right)$, the value of $2 v$ does not reach the theoretical limiting value given by eq $1,1.2$. The linearity of $\ln \alpha_{s}^{2}$ against $\ln M_{w}$ is fairly good above the $\Theta$ temperature, but becomes less satisfactory as the temperature is lowered below the $\Theta$ temperature. At the lowest temperature $\left(25^{\circ} \mathrm{C}\right)$, the value of $2 v$ is less certain, but even the lowest value is much larger than the theoretical limiting value $2 / 3$ given by eq 3 .

The relation $\ln \alpha_{\mathrm{s}}^{2} v s . \ln M_{w}$ is curved below the $\Theta$ temperature. Above the $\Theta$ temperature, the conventional parameter $\tau M^{1 / 2}$ has been used generally. The results rearranged with this parameter are shown in Figure 4. $\alpha_{s}^{2}$ is represented by this

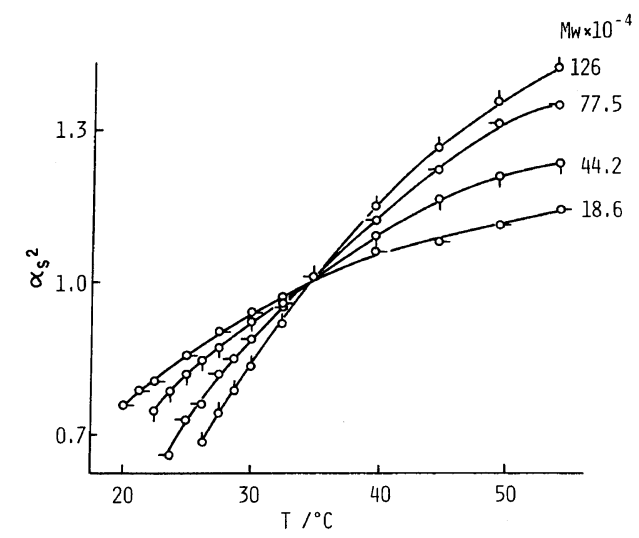

Figure 1. Temperature dependence of $\alpha_{\mathrm{s}}^{2}$ for the polystyrene samples. The molecular weights are indicated in the Figure.

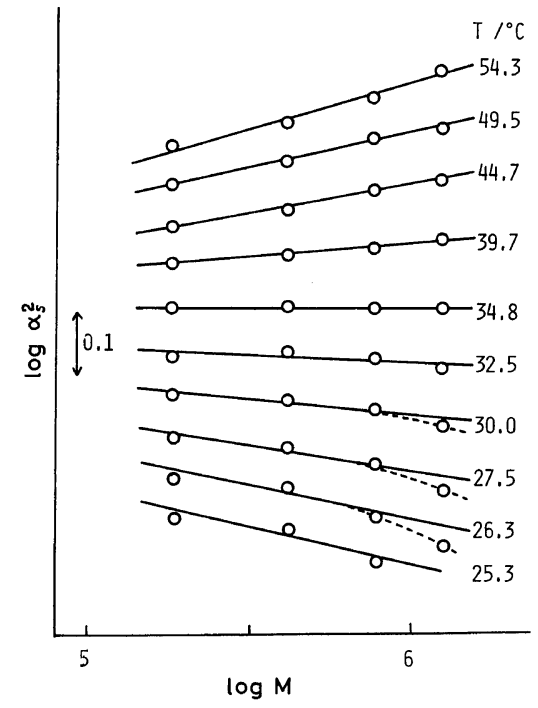

Figure 2. Double-logarithmic plots of $\alpha_{\mathrm{s}}^{2}$ vs. $M_{w}$ around the $\Theta$ temperature.

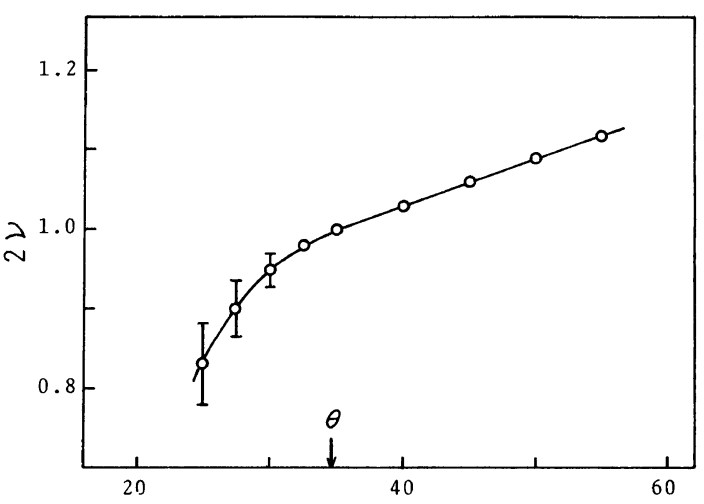

Figure 3. Temperature dependence of the exponent $2 v$ the relation $\left\langle S^{2}\right\rangle \sim M^{2 v}$.

parameter fairly well above and below the $\Theta$ temperature. The curve is smooth and exhibits no sudden change.

The double-logarithmic plot of $\alpha_{\mathrm{s}}^{2} v s .-\tau M^{1 / 2}$ is shown in Figure 5. According to eq 2 and 3, $\alpha_{s}^{2}$ below the $\Theta$ temperarure should theoretically be proportional to $\left(-\tau M^{1 / 2}\right)^{-2 / 3}$. Figure 5 shows that the slope is asymptotically equal to $-2 / 3$. This result indicates the correctness of the theoretical prediction not only on the part of $\tau$ but also on the part of $M$.

Although the confirmation the $\tau$ part has already been given by Nierlich et al., ${ }^{6}$ their data show a 


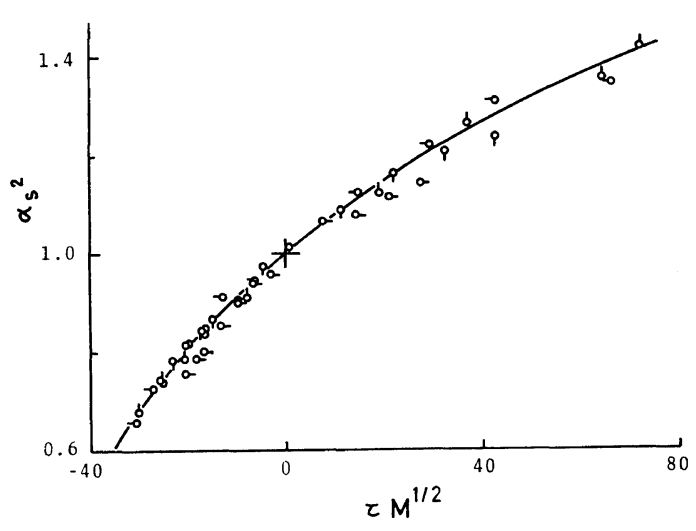

Figure 4. Plot of $\alpha_{\mathrm{s}}^{2} v s . \tau M^{1 / 2}$. Samples are identified in Figure 1.

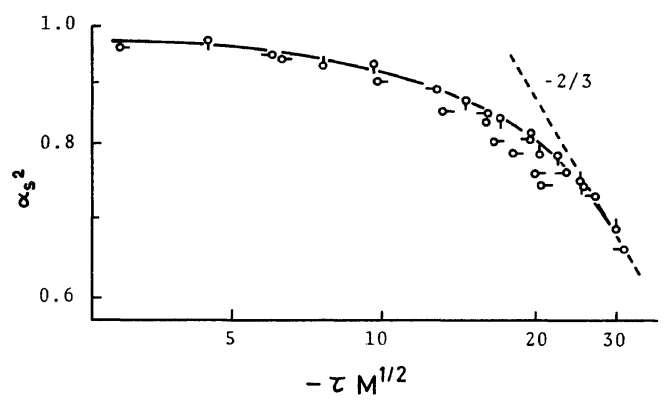

Figure 5. Double-logarithmic plots of $\alpha_{s}^{2} v s .-\tau M^{1 / 2}$. The broken line represents the asymptotic slope $-2 / 3$. relatively sudden change near the "cross-over" point, contrary to our data. Near the cross-over point, the theories ${ }^{1,2}$ have no prediction. However, the change in this region should be smooth. The sudden change observed by Nierlich et al. may be due to the insufficient precision of neutron.

The relation $\alpha_{\mathrm{s}}^{2} \propto M^{2 / 3}$ seems to show the coilglobule transition. However, the values obtained for $\alpha_{\mathrm{s}}^{2}$ are far large compared with that of the "true" compact globule.

\section{REFERENCES}

1. M. Daoud and G. Jannink, J. Phys. (Paris), 37, 973 (1976).

2. T. Oyama and K. Shiokawa, Polym. J., 12, 507 (1980).

3. C. Cuniberti and U. Bianchi, Polymer, 15, 346 (1974).

4. E. Slagowski, B. Tsai, and D. McIntyre, Macromolecules, 9, 687 (1976).

5. R. W. Richards, A. Maconnachie, and G. Allen, Polymer, 19, 266 (1978).

6. M. Nierlich, J. P. Cotton, and B. Farnoux, J. Chem. Phys., 69, 1379 (1978).

7. D. R. Bauer and R. Ullman, Macromolecules, 13, 392 (1980).

8. T. Oyama, K. Shiokawa, and K. Baba, Polym. J., submitted. 